\title{
Late infantile form of multiple sulfatase deficiency
}

\author{
Nami Mohammadian Khonsari(1), Benyamin Hakak-Zargar2 ${ }^{2}$ Tessa Voth ${ }^{3}$ and \\ Shahab Noorian ${ }^{4}$
}

${ }^{1}$ Research Committee, School of Medicine, Alborz University of Medical Sciences, Karaj, Iran, ${ }^{2}$ Faculty of Health Sciences, Simon Fraser University, Burnaby, British Columbia, Canada, ${ }^{3}$ Department of Biomedical Physiology and Kinesiology, Faculty of Science, Simon Fraser University, Burnaby, British Columbia, Canada, and ${ }^{4}$ Department of Pediatrics, School of Medicine, Alborz University of Medical Sciences, Karaj, Iran
Correspondence

should be addressed to N Mohammadian

Khonsari

Email

nami.m.kh@gmail.com

\section{Summary}

Multiple sulfatase deficiency (MSD) is a lysosomal storage disorder (LSD) that results in the accumulation of sulfate esters which go on to cause neurological deterioration and mental delay, skin changes, and dysmorphism. The disease can be categorized into three subtypes based on the age of onset: neonatal, late infantile, or juvenile. Our patient is a 2.5-year-old girl, the only child of a healthy couple. Prior to the presentation of the disease, she had not been noted to have any previous health complications. The condition began at the age of 6 months with developmental regression and global hypotonia. Following thorough evaluation and testing, the patient was diagnosed with severe late infantile MSD, although some features, such as minimal mental deterioration, minimal dysmorphic facial features, and minimal organ enlargement, did not fully correlate with the diagnosis, since in cases of severe forms of the condition these features are almost always quite marked. The unexpected minimalism of some of the patient's MSD signs in spite of the severity of her MSD condition made her case worth further studying.

\section{Learning points:}

- Treating dermatologic signs and symptoms greatly eased our patient's discomfort.

- We would suggest the use of appropriate supportive treatment for symptom management regardless of the life expectancy of the patient.

- As regards the diagnosis of MLD, given that in some cases the patient may present with irregular features of the condition, a genetic evaluation may be useful for accurate diagnosis.

- If motor function impairment is followed by dermatologic involvement, as seen in our patient and in many cases in the literature, MSD must be considered, and additional tests should be done to rule it out.

\section{Background}

Sulfatases are a family of enzymes with the ability to hydrolyze sulfate esters such as steroid sulfates, glycosaminoglycans (GAGs) and sulfolipids (1).

Multiple Sulfatase Deficiency (MSD, OMIM \#272200) is a type of lysosomal storage disorder (LSD) which is inherited through an autosomal recessive (AS) pattern $(2,3)$ that can result in the insufficient activity of all sulfatase family enzymes due to post-translation protein modification defects in which conversion of cysteine into

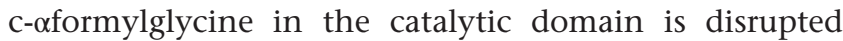
$(4,5)$, thus causing the accumulation of sulfate esters aforementioned $(2,3)$ which results in neurological deterioration and mental delay, organomegaly, skin changes, dysmorphism and skeletal abnormalities (6).

Defects in the SUMF1 gene (OMIM *607939) encoding sulfatase modifying factor 1 (SUMF1) protein 
are responsible for post-translation protein defects (2, 4). More than 30 mutations in SUMF1 gene have been identified, with the majority of them being missense mutations that can disrupt the stability and activity of formylglycine generating enzyme (FGE). Both SUMF1 mutations and altered FGE activity are thought to be involved in the severity of MSD (7). MSD can itself be categorized into three subtypes, depending on the age of disease onset: neonatal, late infantile, or juvenile (8).

The neonatal form is the most severe, resembling mucopolysaccharidosis (MPS) and typically resulting in the death of the patient within 12 months of age $(6,7)$. The late infantile form includes those cases which are severe enough to resemble a late-infantile form of metachromatic leukodystrophy (MLD), which begins within the first year of life with progressive loss of motor and mental abilities and some skeletal changes, but late-infantile MSD can also be mild, with a slow neurodegeneration beginning between the ages of $2-4$ years $(6,7)$. The juvenile subtype is also associated with mental retardation $(6,7)$. These subtype-related phenotypes have no clear correlation to genotypes, and yet nonsense and deletion mutations are expected to cause a severe form of the disease, and in particular, the infantile subtype (5). This disease is very rare, with an estimated prevalence of less than one per million (9), and to our knowledge, after a thorough search through literature, until now, less than 50 cases of MSD have been reported.

\section{Case presentation}

Our patient is a 2.5-year-old girl (at the time of referral), the only child of a healthy non-consanguineous couple. Their families had no history of any genetic disorders. The pregnancy was uneventful and the child was born via a normal delivery at 38.5 weeks with an APGAR score of 9/9 and a birth weight of $3800 \mathrm{~g}$, a length of $49 \mathrm{~cm}$, and a head circumference of $34.5 \mathrm{~cm}$. She had no previous developmental delays nor serious infections. Before the presentation of the disease, medical evaluations where normal, with no neurological impairments and no abnormalities in tendon reflexes seen. At the age of 6 months, she became ill and developmentally regressed, such that she no longer could sit without help and she developed global hypotonia. Thus, clinical investigations began. At first, she was forwarded to the neuro-pediatrician with suspicions of neurodegenerative diseases. Upon neurological evaluation, she avoided eye contact. Reaction to sound stimulus and head control were both absent. Further neurological examination revealed upper and lower tendon hyperreflexia with bilateral upward Babinski signs, with normal vision and eyesight. Thus, a CT scan was obtained from the patient, which revealed the thinning of the corpus callosum and benign hydrocephalus of infancy without white matter changes in the brain. She was subsequently referred to us for endocrine evaluation and assessment of developmental delays. The remarkable observations upon examination of the patient included a number of features of her physical appearance, namely a coarse face, ichthyosis, hypertrichosis. She was hypotonic, and unable to sit and walk on her own (Fig. 1).

\section{Investigation}

The diagnosis of MSD is based on sulfatides and MPS excretion, with multiple sulfatase deficiencies in leukocytes and fibroblasts subsequently confirmed by genetic tests. Since we suspected MSD or MPS as an underlying cause, the following tests were ordered and their results were conclusive. Plasma was tested for VLCFA (very longchain fatty acids). Plasma VLCFA was normal. Blood and urine were tested for arylsulfatase A (ASA) activity, and ASA activity was found to be deficient. Urine showed pathologic sulfatide excretion. This data was compatible with the diagnosis of late-infantile MSD. Thus, a wholeexome sequencing (WES) was ordered. Its platform was illumine HiSeq4000 with a sensitivity of 99\%, and thus the diagnosis was confirmed by DNA analysis. The patient was homozygous for a missense mutation c.739G $>C$ causing a pG247R amino acid substitution in the SUMF1 gene.

After the completion of tests and the acquisition of evidence needed for presenting this case, the presentation

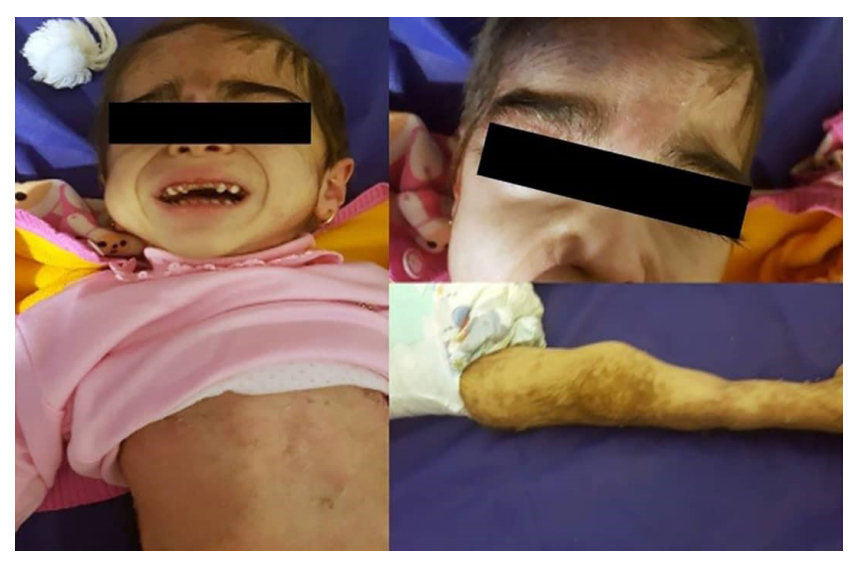

Figure 1

Coarse face, ichthyosis, hypertrichosis. (Guardians permit to publish the photo.) 


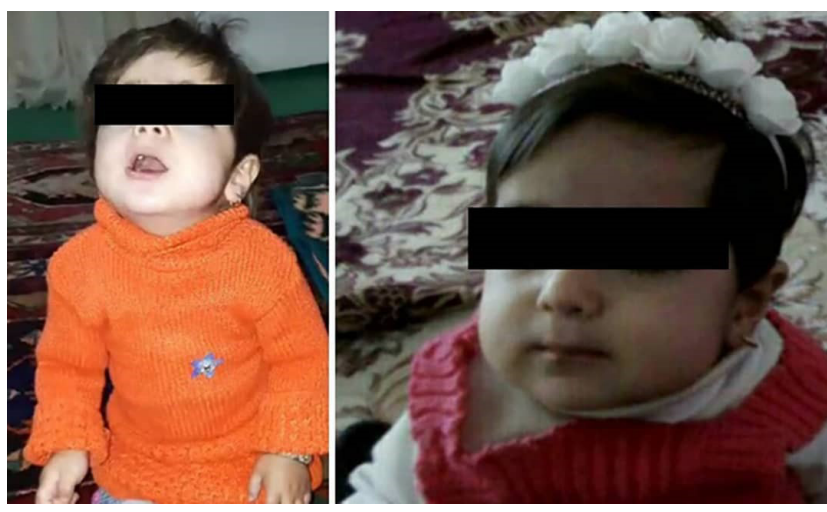

Figure 2

The right picture: before the appearance of the signs and symptoms. The picture on the left shows the first signs and symptoms (hypotonia). (Guardians permit to publish the photo.)

and study were explained to the parents and written consent was obtained from both of them. Afterward, this study was explained to the University's Research Committee and Ethics in Research Committee of Medical Sciences and their approval was obtained.

Sulfatase deficiencies presentations are variable, depending on the enzymes deficient and the genotype. As such, there is variation to the phenotypic presentation of MSD, which is shaped by a combination of isolated sulfatase deficiencies; patients with the disease may present with varying combinations of: coarse facial features, ichthyosis, hepatosplenomegaly, neuropsychomotor retardation, developmental delay, and skeletal changes (10). In this particular case, the features that initially aroused our suspicion of MSD and ultimately led to our diagnosis were the presence of ichthyosis and global developmental delay. In our opinion, this can be a very useful indicator to identify the condition and can be the best diagnostic sign of the disease in infancy. Our patient had typical features of infantile MSD, including hypotonia, coarse face, ichthyosis, visceromegaly, and delayed psychomotor development (Fig. 2). Through studying similar cases mentioned in the literature, we came to the conclusion that the phenotypic outcome depends on both residual FGE activity as well as protein stability, though phenotypes may overlap and the rarity of the disease makes diagnosis challenging. Thus, we propose that a whole-exome sequencing is essential for the diagnosis of MSD.

\section{Treatment}

After the diagnosis, supportive treatment is indicated, since there is no cure for MSD. In our case, we referred our patient to a dermatologist due to dermatologic symptoms (ichthyosis) to treat her by applying one of the many skin softeners available in the market. In our experience, plain petroleum jelly is preferable. We should advise the patients to use these ointments after bathing while the skin is still moist for a better result. Salicylic acid gel and lactate lotion are also effective. In our case, the patient responded extremely well to lactate lotion combined with moisturizing ointment. We did not treat her hypertrichosis, as the treatment could have exacerbated the ichthyosis. In our experience, the best results are acquired when the skin is covered at nights with an airtight, moisturizing ointment. Other treatments should be specific to the management of symptoms, such as orthopedic treatment for curvature of the spine.

\section{Discussion}

What makes this case worth studying was the unusual disparity in the severity of the patient's diagnosis and the presentation of her signs and symptoms. Typically, in a severe case such as our patient's, we would expect to see dermatologic conditions in concomitance with mental retardation, dysmorphism, and organomegaly, as aforementioned. However, in this case, our expectations were supplanted by several findings. First, we noted only minimal mental retardation that was hardly detectable despite all the neurologic conditions. Secondly, her facial dysmorphism was similarly surprising in its minimalism; without the most thorough examination, it could have easily gone undetected. Thirdly, the severity of her organomegaly was likewise unexpectedly mild in comparison to what has been seen in the literature documenting similarly severe cases of MSD. Fourthly, our patient lacked the characteristic demyelination symptoms in the CNS that are typically seen in MLD. Fifthly, there were hydrocephalus and cardiac involvement seen in our patient, which are far less commonly seen than mental retardation or dysmorphism. It was highly unexpected to see cardiac involvement so early when mental regression was at the same time so minimal.

\section{Declaration of interest}

The authors declare that there is no conflict of interest that could be perceived as prejudicing the impartiality of the research reported.

\section{Funding}

This research did not receive any specific grant from any funding agency in the public, commercial or not-for-profit sector. 


\section{Patient consent}

Informed written consent was obtained from the patient's parents and the father's family members regarding their own and their child's clinical details, research findings, and data for publication purposes.

\section{Ethics approval and consent to participate}

Due to the rarity of mutations and the condition of our patient, written informed consent was obtained from her parents to use her data for research purposes. This study was approved by the University and Clinic's Research and Ethics Committees.

\section{Availability of data and materials}

The datasets used and analyzed during the current study are available from the corresponding author in response to reasonable requests and with the permission of the patient's legal guardians.

\section{Author contribution statement}

$\mathrm{N} \mathrm{M} \mathrm{K} \mathrm{H}$ analyzed genetic findings, aided in the genetic study, drafted the initial manuscript, reviewed and revised the manuscript, enrolled the patient in the study, collected the data, and drafted the final manuscript. $\mathrm{B} \mathrm{H} \mathrm{Z}$ and $\mathrm{T} \mathrm{V}$ carried data management, drafted, and revised the final manuscript. S N supervised the project as the principal investigator and revised the final manuscript. All authors approved the final manuscript as submitted and agree to be accountable for all aspects of the work.

\section{Acknowledgement}

We thank all the participants for their cooperation in this project.

\section{References}

1 Dierks T, Dickmanns A, Preusser-Kunze A, Schmidt B, Mariappan M, von Figura K, Ficner R \& Rudolph MG. Molecular basis for multiple sulfatase deficiency and mechanism for formylglycine generation of the human formylglycine-generating enzyme. Cell $2005 \mathbf{1 2 1}$ 541-552. (https://doi.org/110.1016/j.cell.2005.03.001s)

2 Artigalás OA, Da Silva LR, Burin M, Pastores GM, Zeng B, Macedo N \& Schwartz IV. Multiple sulfatase deficiency: clinical report and description of two novel mutations in a Brazilian patient. Metabolic Brain Disease 200924 493-500. (https://doi.org/10.1007/s11011-0099151-8)

3 Mancini GM, van Diggelen OP, Huijmans JG, Stroink H $\&$ de Coo RF. Pitfalls in the diagnosis of multiple sulfatase deficiency. Neuropediatrics 200132 38-40. (https://doi. org/10.1055/s-2001-12213)

4 Dierks T, Schlotawa L, Frese MA, Radhakrishnan K, von Figura K \& Schmidt B. Schmidt BJBeBA-MCR. Molecular basis of multiple sulfatase deficiency, mucolipidosis II/III and Niemann-Pick C1 disease - lysosomal storage disorders caused by defects of nonlysosomal proteins. Biochimica et Biophysica Acta 20091793 710-725. (https://doi.org/10.1016/j.bbamcr.2008.11.015)

5 Schmidt B, Selmer T, Ingendoh A \& von Figurat KJC. A novel amino acid modification in sulfatases that is defective in multiple sulfatase deficiency. Cell 199582 271-278. (https://doi.org/10.1016/00928674(95)90314-3)

6 Schlotawa L, Ennemann EC, Radhakrishnan K, Schmidt B Chakrapani A, Christen HJ, Moser H, Steinmann B, Dierks T \& Gärtner J. SUMF1 mutations affecting stability and activity of formylglycine generating enzyme predict clinical outcome in multiple sulfatase deficiency. European Journal of Human Genetics 2011 19 253-261. (https://doi.org/10.1038/ejhg.2010.219)

7 Schlotawa L, Steinfeld R, von Figura K, Dierks T \& Gartner J. Molecular analysis of SUMF1 mutations: stability and residual activity of mutant formylglycine-generating enzyme determine disease severity in multiple sulfatase deficiency. Human Mutation 200829 205. (https://doi.org/10.1002/humu.9515)

8 Eto Y, Gomibuchi I, Umezawa F \& Tsuda T. Pathochemistry, pathogenesis and enzyme replacement in multiplesulfatase deficiency. Enzyme 198738 273-279. (https://doi. org/10.1159/000469216)

9 Meikle PJ, Hopwood JJ, Clague AE \& Carey WF. Prevalence of lysosomal storage disorders. JAMA 1999281 249-254. (https://doi. org/10.1001/jama.281.3.249)

10 Yiş U, Pepe S, Kurul SH, Ballabio A, Cosma MP \& Dirik EJB. Multiple sulfatase deficiency in a Turkish family resulting from a novel mutation. Brain and Development 200830 374-377. (https://doi. org/10.1016/j.braindev.2007.10.007)

Received in final form 30 July 2020

Accepted 3 September 2020 